\title{
Spectral multipliers for a distinguished Laplacian on certain groups of exponential growth (A remark on the paper of $M$. Cowling et al.)
}

\author{
by \\ AdAm Sikora (Las Cruces, NM)
}

\begin{abstract}
We study spectral multipliers for a distinguished Laplacian on certain groups of exponential growth. We obtain a stronger optimal version of the results proved in $[\mathrm{CGHM}]$ and $[\mathrm{A}]$.
\end{abstract}

The aim of this note is to introduce some small but significant changes to the proof of the spectral multiplier theorem obtained in [CGHM]. These changes allow us to obtain a stronger version of the result obtained in [CGHM]. The required order of differentiability in our version of the spectral multiplier theorem is $1 / 2$ smaller than in [CGHM]. This improvement is significant as our result is optimal in the sense that this order of differentiability cannot be further decreased (see remark to Theorem 1).

Our proof is valid not only for the groups investigated in [CGHM], namely Iwasawa components of noncompact, semisimple Lie groups, but it also works for the groups considered in [A], i.e. solvable extensions of $H$-type groups. Also in the latter case we obtain a stronger optimal version of the result proved in $[\mathrm{A}]$.

To avoid repetition we only describe this part of the proof which is different than in [CGHM]. For a broader discussion of multiplier theorems on groups of exponential growth we refer the reader to [CGHM]. For basic definitions and facts concerning solvable extensions of $H$-type groups see [A].

Before we state our main result we have to introduce some notation. However, for full description of notation which we use we again refer the reader to $[\mathrm{CGHM}]$. Let $G$ denote a connected, noncompact, semisimple Lie group. Write $G=A N K$ for an Iwasawa decomposition of $G$ and $S$ for the solvable group $A N$. We identify $S$ with the symmetric space $G / K$. Under this identification the $G$-invariant measure is the left-invariant measure on $S$ and the Riemannian metric on $G / K$ is a left-invariant metric on $S$. We denote by $n$

2000 Mathematics Subject Classification: 22E30, 43A15, 43A22.

Research supported by KBN 2 P03A 05814. 
the dimension of $S$, by $l$ its real rank and by $\nu$ the pseudo-dimension $2 d+l$, where $d$ is the cardinality $\left|\Sigma_{0}^{+}\right|$of the set of indivisible positive roots.

If $X$ is a left-invariant vector field on $S$ we denote by $\check{X}$ the rightinvariant vector field on $S$ which coincides with $X$ at the identity. We are going to study the distinguished Laplacian on $S$ defined by the formula

$$
\Delta_{S}=-\sum_{i=1}^{n} \check{X}_{i}^{2},
$$

where $X_{1}, \ldots, X_{n}$ is any orthonormal base of the Riemannian metric on $S$. $\Delta_{S}$ does not depend on the particular choice of orthonormal base. Further $\Delta_{S}$ is self-adjoint and positive definite and it admits a spectral decomposition. By the spectral theorem, for any bounded Borel function $m$ the operator

$$
m\left(\Delta_{S}\right)=\int_{0}^{\infty} m(\lambda) d E_{\Delta_{S}}(\lambda)
$$

is well defined and bounded on $L^{2}(S)$. For the definition of a distinguished Laplacian on solvable extensions of $H$-type groups we refer the reader to [A].

We denote by $H^{s}(\mathbb{R})$ the $L^{2}$-Sobolev space of order $s$ on $\mathbb{R}$. If $m$ is a function on $\mathbb{R}_{+}$which is locally in $H^{s}(\mathbb{R})$ we define $\|m\|_{(s)}$ by

$$
\|m\|_{(s)}=\sup _{t \geq 1}\left\|\eta \delta_{t} m\right\|_{H^{s}}
$$

where $\eta \in C_{\mathrm{c}}^{\infty}\left(\mathbb{R}_{+}\right)$is any nonzero smooth function with compact support on $\mathbb{R}_{+}$and $\delta_{t} m(\lambda)=m(t \lambda)$. Note that the condition $\|m\|_{(s)}<\infty$ is actually independent of the choice of $\eta$. The main goal of this paper is to prove the following theorem which is a stronger optimal version of [CGHM, Theorem 2.1] and [A, Theorem 2].

TheOREM 1. Suppose that $L$ is the distinguished Laplacian on an Iwasawa $A N$ group or on a solvable extension of an $H$-type group. Fix $s_{0}$ and $s_{\infty}$ in $\mathbb{R}_{+}$such that

$$
s_{0}>\nu / 2 \quad \text { and } s_{\infty}>\max \{\nu / 2, n / 2\}\left(^{1}\right) .
$$

Let $m$ be a function on $[0, \infty)$ such that

(i) on the interval $[0,2], m$ coincides with a function in $H^{s_{0}}(\mathbb{R})$;

(ii) $m$ is locally in $H^{s_{\infty}}$ and $\|m\|_{\left(s_{\infty}\right)}<\infty$.

Then $m\left(\Delta_{S}\right)$ is bounded on $L^{p}(S)$ for $1<p<\infty$ and is of weak type $(1,1)$.

REMARK. The order of differentiability required in Theorem 1 is optimal. Indeed, let $\sigma^{a}\left(\Delta_{S}\right)=\left(1-\Delta_{S}\right)_{+}^{a}$ be the Riesz mean of $\Delta_{S}$ of order $a$. Then on complex symmetric spaces, $\sigma^{a}\left(\Delta_{S}\right)$ is bounded on all spaces $L^{p}(S)$ if and

$\left({ }^{1}\right) \nu=3$ for any solvable extension of an $H$-type group. 
only if $a>(n-1) / 2=(\nu-1) / 2($ see $[\mathrm{H}])$. Now the function $(1-\lambda)_{+}^{a}$ belongs to $H^{s}$ if and only if $a>s-1 / 2$. Hence Theorem 1 describes the optimal range of summability of Riesz means. Another way to see that the order of differentiability required in Theorem 1 is optimal is to consider imaginary powers of $L$. Indeed, let $\varphi$ be a smooth function equal to 0 on $[0,1]$ and 1 off $[0,2]$. We put $m_{\alpha}(\lambda)=\varphi(\lambda) \lambda^{i \alpha}$. Then (see [SW])

$$
\left\|m_{\alpha}\left(\Delta_{S}\right)\right\|_{L^{1} \rightarrow L^{1, \infty}} \geq C(1+|\alpha|)^{n / 2} .
$$

However, $\left\|m_{\alpha}\right\|_{(s)} \sim(1+|\alpha|)^{s}$ and so the conclusion of Theorem 1 does not hold for any $s_{\infty}<n / 2$.

Proof of Theorem 1. First we note that if (i) and (ii) hold for $m$, then $\widetilde{m}(\lambda)=m(\lambda-1)$ also satisfies (i) and (ii). Hence it is enough to prove that $m\left(\Delta_{S}^{\prime}\right)$ is of weak type $(1,1)$, where $\Delta_{S}^{\prime}=\Delta_{S}+I$. We denote by $K_{m\left(\Delta_{S}^{\prime}\right)}$ the convolution kernel associated with $m\left(\Delta_{S}^{\prime}\right)$ (see [CGHM]). We let $B_{r}$ denote the open ball of radius $r$ centred at the identity of $S$ defined relative to the left-invariant metric. Finally we put $A_{k}=B_{2^{k / 2}} \backslash B_{2^{(k-1) / 2}}$.

A minor variation of the standard Calderón-Zygmund technique described in $[\mathrm{CGHM}]$ reduces the proof of Theorem 1 to the following two propositions (compare [CGHM, Proposition 3.2, Lemma 4.4]):

Proposition 1. Suppose that $\varepsilon$ is a positive real number and $X$ is a leftinvariant vector field on $S$. For all nonnegative integers $j$ and all integers $k$, if $\operatorname{supp} m \subset\left[2^{j-2}, 2^{j}\right]$, then

$$
\begin{aligned}
\left\|\chi_{A_{k}} K_{m\left(\Delta_{S}^{\prime}\right)}\right\|_{L^{2}} & \leq C_{\varepsilon} 2^{n j / 4}\left(2^{k+j}+1\right)^{\varepsilon-s / 2}\left\|\delta_{2^{j}} m\right\|_{H^{s}}, \\
\left\|\chi_{A_{k}} X K_{m\left(\Delta_{S}^{\prime}\right)}\right\|_{L^{2}} & \leq C_{\varepsilon} 2^{(n+2) j / 4}\left(2^{k+j}+1\right)^{\varepsilon-s / 2}\left\|\delta_{2^{j}} m\right\|_{H^{s}} .
\end{aligned}
$$

Proposition 2. Suppose $s>\nu / 2$ and $\operatorname{supp} m_{0} \subset[-1, b]$ where $b \in \mathbb{R}$. Then

$$
\left\|K_{m_{0}\left(\Delta_{S}^{\prime}\right)}\right\|_{L^{1}(S)}=\left\|m_{0}\left(\Delta_{S}^{\prime}\right)\right\|_{L^{1}(S) \rightarrow L^{1}(S)} \leq C_{b}\left\|m_{0}\right\|_{H^{s}} .
$$

Proof of Propositions 1 and 2. First we note that (see [CGHM, (1.1)])

$$
\begin{aligned}
\int_{S}\left|K_{m\left(\Delta_{S}^{\prime}\right)}(g)\right|^{2} d g & =\int_{\mathfrak{a}^{*}}|m(1+\langle\lambda, \lambda\rangle)|^{2}|\mathbf{c}(\lambda)|^{-2} d \lambda \\
& \leq C^{\prime} \int_{0}^{\infty}|m(\tau)|^{2} \tau^{n / 2-1} d \tau=C \int_{\mathbb{R}^{n}}\left|K_{m\left(\Delta_{\left.\mathbb{R}^{n}\right)}\right.}(x)\right|^{2} d x,
\end{aligned}
$$

where $\Delta_{\mathbb{R}^{n}}$ is the Laplace operator on $\mathbb{R}^{n}$. Hence

$$
\left\|\chi_{A_{k}} K_{m\left(\Delta_{S}^{\prime}\right)}\right\|_{L^{2}} \leq\left\|K_{m\left(\Delta_{S}^{\prime}\right)}\right\|_{L^{2}} \leq C 2^{n j / 4}\left\|\delta_{2^{j}} m\right\|_{L^{2}} .
$$

It is proved in [CGHM, Lemma 4.4] that if supp $m \subset\left[2^{j-2}, 2^{j}\right]$, then

$$
\left\|\chi_{A_{k}} K_{m\left(\Delta_{S}^{\prime}\right)}\right\|_{L^{2}} \leq C\left(2^{(1-2 s)(k+j) / 4+n j / 4}+2^{n j / 4} e^{-2^{k+j-4}}\right)\left\|\delta_{2^{j}} m\right\|_{H^{s}} .
$$


By (8) and (7), if supp $m \subset\left[2^{j-2}, 2^{j}\right]$, then

$$
\left\|\chi_{A_{k}} K_{m\left(\Delta_{S}^{\prime}\right)}\right\|_{L^{2}} \leq C 2^{n j / 4}\left(2^{k+j}+1\right)^{1 / 4-s / 2}\left\|\delta_{2^{j}} m\right\|_{H^{s}} .
$$

Now we show that one can put any $\varepsilon>0$ in place of $1 / 4$ in (9). To do this we use the Mauceri-Meda interpolation trick (see $[\mathrm{MM}]$ ). By interpolation between (9) and (7),

$$
\left\|\chi_{A_{k}} K_{m\left(\Delta_{S}^{\prime}\right)}\right\|_{L^{2}} \leq C 2^{n j / 4}\left(2^{k+j}+1\right)^{\theta(1 / 4-s / 2)}\left\|\delta_{2^{j}} m\right\|_{\left[L^{2}, H^{s}\right]_{\theta}} .
$$

Hence by putting $s^{\prime}=\theta s$ and taking $\theta$ small enough we obtain (3).

To prove (4) we note that by (6) for any bounded Borel function $m$,

$$
\begin{aligned}
\left\|X K_{m\left(\Delta_{S}^{\prime}\right)}\right\|_{L^{2}} & \leq C\left(\left\|\check{X} K_{m\left(\Delta_{S}^{\prime}\right)}\right\|_{L^{2}}+\left\|K_{m\left(\Delta_{S}^{\prime}\right)}\right\|_{L^{2}}\right) \\
& \leq C\left\|\sqrt{\Delta_{S}^{\prime}} K_{m\left(\Delta_{S}^{\prime}\right)}\right\|_{L^{2}} \leq C 2^{(n+2) j / 4}\left\|\delta_{2^{j}} m\right\|_{L^{2}} .
\end{aligned}
$$

The rest of the proof of (4) is the same as for (3).

Note that for any $a>0$ the operator $\Delta_{S}+a I$ satisfies the Gaussian bounds for the corresponding heat kernels. It also satisfies the finite speed propagation of the solution of the wave equation (see (16)). We do not use any other properties in the proof of (3). Hence (3) holds for $\Delta_{S}+a I$ and so (3) holds for all functions $m$ such that supp $m \subset\left[-1,2^{j}\right]$. Now Proposition 2 follows from (3). Indeed by [CGHM, Lemma 1.3 and (1.3)] and (3),

$$
\begin{aligned}
& \left\|K_{m_{0}\left(\Delta_{S}^{\prime}\right)}\right\|_{L^{1}(S)}=\left\|\chi_{B_{1}} K_{m_{0}\left(\Delta_{S}^{\prime}\right)}\right\|_{L^{1}}+\sum_{k>0}\left\|\chi_{A_{k}} K_{m_{0}\left(\Delta_{S}^{\prime}\right)}\right\|_{L^{1}} \\
& \quad \leq\left\|\phi_{0} \chi_{B_{1}}\right\|_{L^{2}}\left\|\chi_{B_{1}} K_{m_{0}\left(\Delta_{S}^{\prime}\right)}\right\|_{L^{2}}+\sum_{k>0}\left\|\phi_{0} \chi_{A_{k}}\right\|_{L^{2}}\left\|\chi_{A_{k}} K_{m_{0}\left(\Delta_{S}^{\prime}\right)}\right\|_{L^{2}} \\
& \quad \leq C\left(\left\|\chi_{B_{1}} K_{m_{0}\left(\Delta_{S}^{\prime}\right)}\right\|_{L^{2}}+\sum_{k>0} 2^{k \nu / 4}\left\|\chi_{A_{k}} K_{m_{0}\left(\Delta_{S}^{\prime}\right.}\right\|_{L^{2}}\right) \\
& \quad \leq C\left(\left\|m_{0}\right\|_{L^{2}}+\sum_{k>0} 2^{(\nu-2 s+\varepsilon) k / 4}\left\|m_{0}\right\|_{H^{s}}\right) \leq C\left\|m_{0}\right\|_{H^{s}}
\end{aligned}
$$

This ends the proof of Propositions 1 and 2 and Theorem 1.

Finally we describe an alternative way of proving Propositions 2 and 1. This way is independent of [CGHM, Lemma 4.4] (we do not need (8) in the proof). In contrast to [A, CGHM], in this proof we do not need estimates for the gradient of the heat kernel. Actually the result which we obtain is more precise than Propositions 2 and 1.

Proposition 3. Suppose that $\operatorname{supp} m_{0} \subset[-1, b]$ where $b \in \mathbb{R}_{+}$. Then

$$
\left\|K_{m_{0}\left(\Delta_{S}^{\prime}\right)}\right\|_{L^{1}(S)}=\left\|m_{0}\left(\Delta_{S}^{\prime}\right)\right\|_{L^{1}(S) \rightarrow L^{1}(S)} \leq C_{b}\left\|m_{0}\right\|_{B_{\nu / 2}^{2,1}},
$$

where $B_{s}^{q, q^{\prime}}$ denotes the Besov space (see $[\mathrm{T}]$ for definition and basic properties). Moreover if $k \in \mathbb{Z}$ and $j \in \mathbb{Z}_{+}$and $\operatorname{supp} m \subset\left[0,2^{j}\right]$, then 


$$
\begin{aligned}
&\left\|\chi_{A_{k}} K_{m\left(\Delta_{S}^{\prime}\right)}\right\|_{L^{2}} \leq C 2^{n j / 4}\left(2^{k+j}+1\right)^{-s / 2}\left\|\delta_{2^{j}} m\right\|_{B_{s}^{2, \infty}}, \\
&\left\|\chi_{A_{k}} X K_{m\left(\Delta_{S}^{\prime}\right)}\right\|_{L^{2}} \leq C 2^{(n+2) j / 4}\left(2^{k+j}+1\right)^{-s / 2}\left\|\delta_{2^{j}} m\right\|_{B_{s}^{2, \infty}} .
\end{aligned}
$$

Proof. By (6),

$$
\int_{S}\left|K_{m\left(\Delta_{S}^{\prime}\right)}(g)\right|^{2} d g \leq C \int_{\mathbb{R}^{n}}\left|K_{m\left(\Delta_{\mathbb{R}^{n}}\right)}(x)\right|^{2} d x .
$$

Next, if $b_{r}$ is the Euclidean ball of radius $r$ centred at 0 in $\mathbb{R}^{n}$ and $\chi_{b_{r}}$ is the characteristic function of $b_{r}$, then the functions $\chi_{b_{r}} K_{m\left(\Delta_{\mathbb{R}} n\right)}$ and $\left(1-\chi_{b_{r}}\right) K_{m\left(\Delta_{\mathbb{R}^{n}}\right)}$ are radial, so there exist functions $m_{r}: \mathbb{R}_{+} \rightarrow \mathbb{C}$ and $m^{r}: \mathbb{R}_{+} \rightarrow \mathbb{C}$ such that

$$
K_{m_{r}\left(\Delta_{\mathbb{R}} n\right)}=\chi_{b_{r}} K_{m\left(\Delta_{\mathbb{R}} n\right)} \quad \text { and } \quad K_{m^{r}\left(\Delta_{\mathbb{R}^{n}}\right)}=\left(1-\chi_{b_{r}}\right) K_{m\left(\Delta_{\mathbb{R}} n\right)} .
$$

Now we put $M(\lambda)=m_{r}\left(\lambda^{2}\right)$ and we denote by $\widehat{M}$ the Fourier transform of $M$. Then supp $\widehat{M} \subset[-r, r]$. By the finite speed propagation property of the wave equation (cf. [S, Theorem 1]), we have

$$
\operatorname{supp}\left(K_{C_{t}\left(\sqrt{\Delta_{S}^{\prime}}\right)}\right) \subset B_{t},
$$

where $C_{t}(\lambda)=\cos (t \lambda)$. Hence using the functional calculus formula

$$
m_{r}\left(\Delta_{S}^{\prime}\right)=M\left(\sqrt{\Delta_{S}^{\prime}}\right)=\frac{1}{2 \pi} \int \widehat{M}(t) C_{t}\left(\sqrt{\Delta_{S}^{\prime}}\right) d t
$$

we obtain

$$
\operatorname{supp}\left(m_{r}\left(\Delta_{S}^{\prime}\right)\right) \subset B_{r}
$$

Obviously $m=m_{r}+m^{r}$ so by (17),

Hence by (15),

$$
K_{m^{r}\left(\Delta_{S}^{\prime}\right)}(g)=K_{m\left(\Delta_{S}^{\prime}\right)}(g) \quad \text { for } g \notin B_{r} .
$$

$$
\begin{aligned}
\int_{B_{r}^{c}}\left|K_{m\left(\Delta_{S}^{\prime}\right)}(g)\right|^{2} d g & =\int_{B_{r}^{c}}\left|K_{m^{r}\left(\Delta_{S}^{\prime}\right)}(g)\right|^{2} d g \leq \int_{S}\left|K_{m^{r}\left(\Delta_{S}^{\prime}\right)}(g)\right|^{2} d g \\
& \leq C \int_{\mathbb{R}^{n}}\left|K_{m^{r}\left(\Delta_{\mathbb{R}^{n}}\right)}(x)\right|^{2} d x=C \int_{b_{r}^{c}}\left|K_{m\left(\Delta_{\left.\mathbb{R}^{n}\right)}\right.}(x)\right|^{2} d x .
\end{aligned}
$$

Thus

$$
\begin{aligned}
\left\|\chi_{A_{k+1}} K_{m\left(\Delta_{S}^{\prime}\right)}\right\|_{L^{2}} & \leq\left\|\chi_{B_{2^{k / 2}}^{\mathrm{c}}} K_{m\left(\Delta_{S}^{\prime}\right)}\right\|_{L^{2}} \leq C\left\|\chi_{b_{2^{k} / 2}^{\mathrm{c}}} K_{m\left(\Delta_{\mathbb{R}} n\right)}\right\|_{L^{2}} \\
& \leq C 2^{n j / 4}\left(2^{k+j}+1\right)^{-s / 2}\left\|\delta_{2^{j}} m(|\cdot|)\right\|_{B_{s}^{2, \infty}\left(\mathbb{R}^{n}\right)} \\
& \leq C 2^{n j / 4}\left(2^{k+j}+1\right)^{-s / 2}\left\|\delta_{2^{j}} m\right\|_{B_{s}^{2, \infty}(\mathbb{R})} .
\end{aligned}
$$

To prove (14) we note that (see (10))

$$
\left\|X K_{m\left(\Delta_{S}^{\prime}\right)}\right\|_{L^{2}} \leq C\left\|\sqrt{\Delta_{S}^{\prime}} K_{m\left(\Delta_{S}^{\prime}\right)}\right\|_{L^{2}} \leq\left\|K_{m\left(\Delta_{\mathbb{R}^{n+2}}\right)}\right\|_{L^{2}} .
$$

Now the rest of the proof of (14) is the same as the proof of (13). 
Finally (see (11)),

$$
\begin{aligned}
& \left\|K_{m_{0}\left(\Delta_{S}^{\prime}\right)}\right\|_{L^{1}(S)} \leq C\left(\left\|\chi_{B_{1}} K_{m_{0}\left(\Delta_{S}^{\prime}\right)}\right\|_{L^{2}}+\sum_{k>0} 2^{k \nu / 4}\left\|\chi_{A_{k}} K_{m_{0}\left(\Delta_{S}^{\prime}\right)}\right\|_{L^{2}}\right) \\
& \leq C\left(\left\|K_{m_{0}\left(\Delta_{S}^{\prime}\right)}\right\|_{L^{2}}+\sum_{k \geq 0} 2^{k \nu / 4}\left\|\chi_{B_{2}^{\mathrm{c}} / 2} K_{m_{0}\left(\Delta_{S}^{\prime}\right)}\right\|_{L^{2}}\right) \\
& \leq C\left(\left\|K_{m_{0}\left(\Delta_{\mathbb{R}^{n}}\right)}\right\|_{L^{2}}+\sum_{k \geq 0} 2^{k \nu / 4}\left\|\chi_{b_{2^{k} / 2}^{\mathrm{c}}} K_{m_{0}\left(\Delta_{\mathbb{R}^{n}}\right)}\right\|_{L^{2}}\right) \\
& =C\left(\left\|K_{m_{0}\left(\Delta_{\mathbb{R}^{n}}\right)}\right\|_{L^{2}}+\sum_{k \geq 0} \sum_{i>k} 2^{k \nu / 4}\left\|\chi_{a_{i}} K_{m_{0}\left(\Delta_{\mathbb{R}^{n}}\right)}\right\|_{L^{2}}\right) \\
& \leq C\left(\left\|K_{m_{0}\left(\Delta_{\mathbb{R}} n\right)}\right\|_{L^{2}}+\sum_{i>0} 2^{i \nu / 4}\left\|\chi_{a_{i}} K_{m_{0}\left(\Delta_{\mathbb{R}} n\right)}\right\|_{L^{2}}\right) \\
& =C\left\|m_{0}(|\cdot|)\right\|_{B_{\nu / 2}^{2,1}\left(\mathbb{R}^{n}\right)} \leq C\left\|m_{0}\right\|_{B_{\nu / 2}^{2,1}(\mathbb{R})},
\end{aligned}
$$

where $a_{k}=b_{2^{k / 2}} b_{2^{(k-1) / 2}}$.

\section{References}

[A] F. Astengo, Multipliers for a distinguished Laplacean on solvable extensions of H-type groups, Monatsh. Math. 120 (1995), 179-188.

[CGHM] M. Cowling, S. Giulini, A. Hulanicki, and G. Mauceri, Spectral multipliers for a distinguished Laplacian on certain groups of exponential growth, Studia Math. 111 (1994), 103-121.

$[\mathrm{H}] \quad$ W. Hebisch, The subalgebra of $L(A N)$ generated by the Laplacian, Proc. Amer. Math. Soc. 117 (1993), 547-549.

[MM] G. Mauceri and S. Meda, Vector-valued multipliers on stratified groups, Rev. Mat. Iberoamericana 6 (1990), 141-154.

[S] A. Sikora, On-diagonal estimates on Schrödinger semigroup kernels and reduced heat kernels, Comm. Math. Phys. 188 (1997), 233-249.

[SW] A. Sikora and J. Wright, Imaginary powers of Laplace operators, Proc. Amer. Math. Soc. 129 (2001), 1745-1754.

[T] H. Triebel, Theory of Function Spaces. III, Birkhäuser, Basel, 1992.

Department of Mathematical Sciences

New Mexico State University

P.O. Box 30001

Las Cruces, NM 88003-8001, U.S.A.

E-mail: asikora@nmsu.edu

Received on March 22, 2001;

revised version on February 5, 2002 